The whole account will well repay a careful perusal, and anyone engaged on the design of a flying machine will find much useful information in the results of the various experiments on bodies rotating in a current of air.

\section{THE MEXICAN EARTHQUAKE.}

$A$ NOTHER great earthquake has been added to A the series which has marlked the recent increase in seismic and volcanic activity along the Pacific coast of America. At I I.30 p.m. on Sunday, April I4, or about $6 \mathrm{a} . \mathrm{m}$. of April ${ }_{15}$ by Greenwich time, the greater part of Mexico was visited by a destructive earthquake. As usual, the first accounts were not only exaggerated, but gave an erroneous impression of the distribution of damage; Mexico city, which was represented as almost destroyed, proved by later accounts to have been comparatively little damaged; while the towns of Chilpancingo and Chilapu, as well as some others not to be found in ordinary atlases, suffered great destruction. The sea-coast towns from Salina Cruz to Acapulco suffered severely, and a portion of the latter is said to have been submerged. The shock is reported as severe at San Luis Potori and Juan Batista, though no damage was done at either place; these two cities are about 530 miles apart and about $35^{\circ}$ miles from the region of greatest damage, so we may estimate the area over which the shock was sensible as extending to somewhere about 500 miles from the centre of the disturbance.

The earliest reports stated that railway communication between Mexico city and Vera Cruz was suspended owing to the sinking of the permanent way, but this news, which has not been corroborated in later telegrams, is the only suggestion that the focus of the earthquake may have extended to any distance from the west coast. Everything else points to the conclusion that it originated close to the shore-line of the Pacific, and was partly, if not wholly, submarine. Sea-quakes are common in this region; sometimes they are felt by ships at sea though unnoticed on shore, and in at least one instance seem to have caused the loss of a ship. The story is a remarkable one. On October 3, I902, the German barque Freya cleared from Manzanillo for Panta Arenas; nothing more has been heard of the captain or crew, but the ship was found, twenty days later, partially dismasted and lying on its side. There was nothing to explain the condition of the ship, but a wall calendar in the captain's cabin showed that the catastrophe must have overtaken it on October 4, not long after leaving port, as was also indicated by the anchor being found still hanging free at the bow. Weather reports show that only light winds were experienced in this region from October 3 to October 5, but, on the other hand severe earthquakes were felt at Acapulco and Chilpanzingo on October 4 and 5 , one of which probably caused the damage to the Freya which led to its abandonment.

Prominence has been given in the daily papers to earthquakes in Spain and Italy, which occurred shortly after the Mexican one; but they were of an arder the occurrence of which is too frequent to justify any direct connection between them and the greater one. It may be different as regards the other two large earthquakes, which were registered at 9. Io p.m. on April I8, and at oh. II a.m. on April 19; no news of these shocks has yet reached us; they must have been earthquakes of the first order of importance, but are only known from distant records, which are interpreted as showing that they originated at about $90^{\circ}$ from western Europe. This is about the distance of Mexico, but it is rare for after- shocks to be of as great magnitude as these; on the other hand, it is not uncommon for earthquakes to take place in groups, usually originating at nearly opposite points in the globe. We may consequently, in the absence of news of a great earthquake in America or Japan, look for the origin of these two earthquakes in the North Pacific Ocean on the eastern part of the Malay Peninsula.

\section{TUBERCULOSIS RESEARCH AND \\ VIVISECTION.}

THE investigations conducted by the Royal Commission on 'Tuberculosis, contained in a second interim report recently issued, ${ }^{1}$ would have been impossible without the use of experiments on animals, and the appearance of this report is most opportune, for, almost simultaneously, the Royal Commission on Vivisection has published the first volume of the minutes of evidence taken before it.

As regards the investigations on tuberculosis, thirty different viruses isolated from cases of tuberculosis occurring spontaneously in bovines have been studied, and the results of introducing them into a number of different animals by feeding and by inoculation are recorded. In calves, inoculation usually results in generalised progressive tuberculosis, but the effect is somewhat dependent on the dose, i.e. the number of bacilli, administered. Feeding, on the other hand, usually produces lesions limited to the neighbourhood of the digestive tract, which generally re'rogress and become calcareous. The bovine bacillus, when introduced into rhesus monkeys or chimpanzees either by inoculation or by feeding, induces rapid generalised tuberculosis, and considering the close relation that exists between the anthropoid apes and man, these results are of the highest importance. In pigs generalised progressive tuberculosis is readily set up both by feeding with, and by the inoculation of, bovine bacilli. Goats, dogs, and cats are relatively less susceptible, but more or less tuberculous infection can similarly be produced in them. On this part of the investigation the commissioners remark that the bacillus of bovine tuberculosis is not so constituted as to act on bovine tissues only, and the fact that it can readily infect the anthropoid apes, and, indeed, seems to produce this result more readily than in the bovine body itself, has an importance so obvious that it need not be dwelt on.

The viruses isolated from sixty cases of the disease in man have also been studied, and the results obtained show that they may be divided into two groups, subsequently referred to as group i. and group ii. The bacilli of group i. were mostly obtained from cases of abdominal tuberculosis occurring in children, and the results produced by introducing them into animals are identical with those produced by the bovine bacillus. 'The bacilli of group ii., obtained from various forms of human tuberculosis, grow more luxuriantly in culture than those of group i., and inoculated into calves and rabbits do not produce the generalised and fatal disease caused by the bovine bacillus, but in rhesus monkeys and in the chimpanzee set up a general tuberculosis. Certain human viruses, differing in certain respects from those of groups i. and ii., were also met with, and are classed as group iii., but an opinion on their significance is reserved for a future report.

The commissioners conclude that the tubercle bacillus in its nutritive and reproductive powers re1 Second Interim Report of the IRoyal Commission appointed to inquire into the Relations of Human and Animal Tuberculosis, Part i., Report. Part ii Appendix. "Vol. iv., "Comparative Histofogical and Bacterio logical Investigations." By Dr. Arthur Eastwood. 